DOI: $10.35381 /$ r.k.v4i1.372

\title{
Evaluación de Control Interno en Riesgos Estratégicos para la Dirección de Planificación de la Universidad de Cuenca
}

\section{Evaluation of Internal Control in Strategic Risks for the Planning Department of the University of Cuenca}

\author{
Karla Johanna Argudo Tello \\ kargudot@psg.ucacue.edu.ec \\ Universidad Católica de Cuenca \\ Ecuador \\ https://orcid.org/0000-0001-6129-0409 \\ Juan Carlos Erazo Álvarez \\ jcerazo@ucacue.edu.ec \\ Universidad Católica de Cuenca \\ Ecuador \\ https://orcid.org/0000-0001-6480-2270 \\ Cecilia Ivonne Narváez Zurita \\ inarvaez@ucacue.edu.ec \\ Universidad Católica de Cuenca \\ Ecuador \\ https://orcid.org/0000-0002-7437-9880
}

Recibido: 10 de agosto de 2019

Aprobado: 30 de agosto de 2019

\section{RESUMEN}

El proceso de control en la administración pública, está supeditado a una serie de normativas, siendo responsabilidad de la máxima autoridad orientar las actividades que posibiliten su diseño, implementación y funcionamiento. El propósito de esta investigación es establecer de qué manera las evaluaciones de control interno, enfocadas en riesgos estratégicos, aportan como una herramienta para el cumplimiento de los objetivos institucionales, a través de la Dirección de Planificación de la Universidad de Cuenca. Se realizó un estudio analítico-sintético, basado en un enfoque mixto, para lo cual se diseñó un cuestionario de control interno con preguntas orientadas al componente 
"evaluación de riesgos", del cual se obtuvo como resultado que, la Dirección de Planificación, encargada de la coordinación del sistema de planificación institucional, si bien desarrolla procedimientos que incluyen objetivos a corto y largo plazo, no cuenta con un sistema de evaluación de control interno para gestionar potenciales riesgos.

Descriptores: Evaluación; Control interno; Riesgos estratégicos; Objetivos.

\begin{abstract}
The control process in the public administration is subject to set of regulations, being the responsibility of the highest authority to guide the activities that enable its design, implementation and operation. The purpose of this research is to establish how internal control evaluations, focused on strategic risks, contribute as a tool for the fulfillment of institutional objectives, through the Planning Direction of the University of Cuenca.

An analytical-synthetic study was conducted, based on a mixed approach, for which an internal control questionnaire was designed with questions oriented to the "risk assessment" component, which resulted in the Planning Direction, in charge of coordinating the institutional planning system, although it develops procedures that include short and long-term objectives, it does not have an internal control evaluation system to manage potential risks.
\end{abstract}

Descriptors: Evaluation; Internal control; Strategic risks; Objectives.

\title{
INTRODUCCIÓN
}

Evaluar el cumplimiento de los objetivos, así como la gestión eficiente y eficaz de los recursos que se asignan a las universidades públicas en el Ecuador, es una terea indispensable; dicha evaluación, se basa en una correcta definición y aplicación de controles internos, que determinen a su vez, el cumplimiento en materia legal. Uno de los principales inconvenientes es que, si bien las instituciones proporcionan información de su gestión, dicha información suele no ser oportuna ni apropiada, lo que conduce al incumplimiento de sus objetivos (Medina, Lascano, y Neyfe, 2017).

En el Ecuador, la Secretaría de Educación Superior, Ciencia, Tecnología, Innovación y Saberes Ancestrales (SENESCYT), tiene como misión la conducción de la educación superior. De acuerdo con el resumen ejecutivo, presentado en el boletín analítico de la SENESCYT en diciembre de 2018, la ejecución presupuestaria de las instituciones de 
educación superior públicas, en el año 2018, fue de 1.199 millones de USD y un gasto corriente ejecutado de 59,92 millones de USD.

En la provincia del Azuay existe únicamente una universidad pública, tres universidades cofinanciadas y una autofinanciada; constan también 2 institutos técnicos y tecnológicos públicos y 5 particulares. En el año 2018, en el Azuay existieron 45.098 personas matriculadas en universidades y escuelas politécnicas y 3.515 matriculados en institutos técnicos y tecnológicos (SENESCYT, 2018).

En este contexto, la Universidad de Cuenca tiene como misión formar profesionales comprometidos. Su sede se encuentra en la ciudad de Cuenca y se rige por los principios establecidos en la Constitución de la República y en la Ley Orgánica de Educación Superior. Actualmente, es la única universidad pública en la provincia del Azuay (Universidad de Cuenca, 2018).

En noviembre de 2013, tras las evaluaciones efectuadas por el Consejo de Aseguramiento de la Calidad de la Educación Superior (CEAACES), se determinó que los indicadores académicos de la Universidad de Cuenca, no correspondían a la categoría $A$, debido a falencias en temas como: investigación, número reducido de publicaciones, deficiente infraestructura, entre otros aspectos; sin embargo, en el año 2016 retornó a la categoría A, gracias a su mayor participación en investigación científica, publicaciones, implementación de laboratorios, renovación de planta docente y tecnología (El Telégrafo E.P., 2016).

Actualmente, las publicaciones indexadas, el reconocimiento internacional y la difusión de proyectos, ubican a la Universidad de Cuenca en el quinto lugar entre las mejores universidades del país, y en primer puesto de las universidades públicas del Ecuador (El Telégrafo E.P., 2018).

No obstante, el problema detectado en la presente investigación, es el escaso desarrollo y aplicación de mecanismos, por parte de la Dirección de Planificación de la Universidad de Cuenca, que permitan evaluar el control interno, con relación a los riesgos estratégicos internos y externos a los que la Institución está expuesta para el logro de sus objetivos, lo que ha ocasionado que sus dependencias se vean afectadas, tanto en la ejecución y 
resultados de sus procesos operativos, como en sus procesos administrativos y financieros.

Dentro de esta perspectiva, el motivo de desarrollo del trabajo investigativo, se centra en la posibilidad de que pueda ser puesto a consideración de la Dirección de Planificación de la Universidad de Cuenca, como referente de la necesidad de existencia de un sistema de evaluación de control interno para el tratamiento de los riesgos estratégicos a los que la Institución está expuesta y que pudiesen afectar a la consecución de sus objetivos. Vinculado al motivo de la investigación, según dispone la Contraloría General del Estado (2009), dentro del grupo de normas 100-01, el control interno es un proceso que debe ser aplicado por todos los servidores de la institución, el cual genera seguridad razonable en cuanto al logro de los objetivos. Constituyen elementos de control interno: el ambiente de control, la evaluación de riesgos, las actividades de control, los sistemas de información y comunicación; $y$, el seguimiento.

En concordancia con lo descrito, Rivas (2011), en base a lo que contempla el modelo coso, refiere que el control interno comprende cinco fases que se encuentran interrelacionadas y deben ser aplicadas de manera continua a fin de obtener seguridad razonable de que con su aplicación, los objetivos se cumplirán.

A continuación, se detallan los cinco componentes:

\section{Ambiente de control}

Comprende las acciones y circunstancias que conforman el sistema de control en una organización; determina las políticas de comportamiento del personal respecto del control y la manera en la que se estructuran las actividades.

\section{Evaluación de los Riesgos}

Proceso mediante el cual se identifican, analizan y administran los riesgos y las consecuencias de los mismos. De esta forma, el primer paso para la evaluación de los 
riesgos consiste en establecer los objetivos, a fin de que, sobre esta base, se puedan identificar los riesgos y realizar un control interno eficaz.

\section{Actividades de Control}

Son las acciones y directrices administrativas que se llevan a cabo a fin de lograr una gestión eficiente y eficaz que contribuyan a asegurar que se están cumpliendo los objetivos trazados y sean un apoyo importante a la gerencia. Las actividades de control incluyen revisiones de alto nivel, en cuanto a resultados versus presupuesto, pronóstico, informes de evaluaciones de desempeño, controles físicos de inventarios, etc. (Castillo, Erazo, Narváez, y Torres, 2019).

\section{Información y comunicación}

De este componente se desprenden documentos con información operacional, administrativa y financiera, relacionada con los resultados del cumplimiento de las metas y objetivos, misma que resulta indispensable al momento de tomar decisiones. La comunicación debe darse de manera amplia, es decir, a todos los niveles de la organización.

\section{Supervisión}

Consiste en la evaluación continua con el fin de determinar si los controles que se están aplicando son adecuados, o si por el contrario, es necesario modificarlos, de acuerdo a las condiciones actuales para asegurar su efectividad. 
Componentes del sistema de control interno, según el modelo COSO 2013 Figura 1.

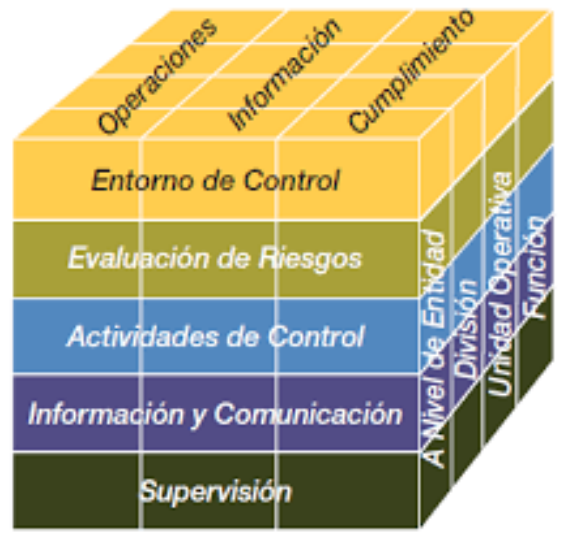

Por otra parte, la Norma de Control Interno 300, de la Contraloría General del Estado (2009) establece que el riesgo es la probabilidad de ocurrencia de un evento no deseado que podría afectar adversamente a la organización. Todo el personal que conforma una institución, será responsable de administrar los riesgos; es decir, aplicar metodologías y procedimientos, a través de los cuales se identificarán, analizarán y tratarán los potenciales acontecimientos que pudiesen interferir en el logro de sus objetivos.

Según la Norma Internacional ISO 31000 (2009), el riesgo es "el efecto de la incertidumbre sobre los objetivos. Se trata, en esencia, circunstancias, sucesos o eventos adversos que impiden el normal desarrollo de las actividades de una empresa y que, en general, tienen repercusiones económicas para sus responsables" (p.4).

\section{Tipos de riesgos}

Cada empresa, según sus condiciones, puede afrontar riesgos diferentes, ya sea interna o externamente.

Los Riesgos Estratégicos hacen referencia a situaciones inesperadas que pueden invalidar la implementación de una decisión estratégica y amenazar la posición competitiva o el logro de las metas estratégicas de una empresa (Owen, 2014).

Seco (2007) afirma: "Los Riesgos Financieros podrían definirse de manera genérica como aquellos derivados de la contingencia o probabilidad de incurrir en una pérdida 
patrimonial como resultado de una transacción financiera o bien por mantener un desequilibrio o posición entre determinados activos y pasivos" (p.6).

Los riesgos financieros pueden ser: Riesgo de Mercado, Riesgo de Crédito y Riesgo de Liquidez (Asociación Española para la Calidad, 2018).

En cuanto a la clasificación de los riesgos, García y Salazar (2015) señalan: Los Riesgos de Operaciones se presentan de la posibilidad que una organización incurra en pérdidas inesperadas, como consecuencia de errores humanos, sistemas de control inadecuados, incumplimiento de controles internos o acontecimientos externos que impidan asegurar la integridad, efectividad y eficiencia de sus operaciones. El Riesgo Legal es aquel que se presenta a causa del incumplimiento de normas, regulaciones y obligaciones contractuales.

El Comité de Supervisión Bancaria de Basilea (2003) define al Riesgo Operativo como aquel que ocasiona pérdidas por factores como: procesos internos inadecuados; inapropiada actuación del personal; y, deficiencia en los sistemas internos, generados por eventos externos; es decir, se asocia a errores humanos, sistemas y procedimientos. El Riesgo Puro consiste en una pérdida, calificada como perjuicio o siniestro, mientras que el Riesgo Especulativo puede tener una incidencia positiva o negativa; es decir, puede resultar en pérdida o ganancia (Gil, 2009).

\section{Técnicas para la identificación de riesgos}

Existe un sinnúmero de herramientas y metodologías para identificar riesgos; sin embargo, para ponerlas en práctica, dependerá de factores como la disponibilidad de recursos económicos, humanos y tecnológicos, pero también se pueden utilizar técnicas combinadas, dependiendo del tipo o de la complejidad de los riesgos. Lo más importante al aplicar una técnica o método es la consistencia en su aplicación, lo cual hará posible que se estandaricen los procesos.

Entre algunas de las técnicas utilizadas constan:

Lluvia de ideas: Castañeda (s.f.) indica que esta técnica consiste en que un grupo de personas, dirigidas por un facilitador, aportan con ideas espontáneas para la solución de 
un problema específico. Las ventajas y desventajas de esta técnica, según lo descrito por el Instituto Nacional de Normalización - INN (2013) son:

Ventajas:

- Estimula la imaginación para identificar nuevos riesgos y sus posibles soluciones.

- Fomenta la comunicación global dentro de la organización.

Desventajas:

- Los participantes pueden no contar con los conocimientos técnicos necesarios para ser colaboradores estratégicos.

- Al ser poco estructurada, es difícil determinar si se ha completado el proceso de identificación de todos los potenciales riesgos.

- Los grupos a menudo impiden la generación de ideas.

Análisis causa-efecto: Método estructurado, con el cual se identifican las causas de un problema; consiste en organizar en categorías, a través de un diagrama de espina de pescado o de un diagrama de árbol, los posibles factores contributivos, de manera que se contemplen todas las hipótesis posibles (Instituto Nacional de Normalización - INN, 2013).

Ventajas:

- Se toman en cuenta todas las hipótesis probables.

- Ilustración gráfica y fácil de entender.

Desventajas:

- No es un proceso completo, necesita ser parte de un análisis de raíz para poder generar recomendaciones.

Lista de chequeo y cuestionarios: Esta técnica utiliza una lista de situaciones o de aspectos de control que se deben comprobar para verificar que no se producen errores significativos en la ejecución de las actividades.

Ventajas:

- Es sencillo de aplicar a múltiples tareas. 
Desventajas:

- Si se confía demasiado en las listas, se deja de realizar análisis exhaustivos de los riesgos de cada actividad.

- Resulta útil para procedimientos repetitivos o estandarizados pero falla cuando intervienen procesos de innovación o adaptación (Delgado, 2016).

Entrevista: Consiste en la interacción dinámica entre el comunicador y el entrevistado. Constituye la primera aproximación a cualquier problema que se desea analizar, especialmente cuando no se tiene información previa del mismo, pero existe una persona involucrada y con conocimiento necesario.

Ventajas:

- Ausencia de presión, de manera que cada entrevistado revela información más precisa, sin que exista influencia o imposición de muchas personas.

Desventajas:

- La validez de los resultados y la calidad de la información recogida depende directamente de la habilidad del entrevistador (Trespalacios, Vázquez y Bello, 2005).

Flujograma: La Asociación Española para la Calidad (2012) refiere que esta herramienta consiste en la representación gráfica de un proceso; muestra paso a paso la secuencia de actividades a realizar para obtener un resultado. Sirve para identificar los problemas y riesgos existentes, dónde y cuándo establecer indicadores y correcciones.

Ventajas:

- Se identifican los pasos, los conflictos de autoridad, las responsabilidades, los cuellos de botella y los puntos de decisión.

- Se pueden identificar claramente las responsabilidades y la interacción de las áreas que intervienen en el mismo.

Desventajas:

- Limita la cantidad de información que se puede ingresar dentro del flujo de proceso.

Análisis de modo y efecto de falla (AMEF): Se refiere al estudio de los efectos sobre diferentes modos de fallas en los componentes de un sistema o en los procesos; se basa 
en un listado, lo más detallado, de las fallas que puede presentar cada uno de dichos procesos.

Ventajas:

- Aplicable a sistemas, procesos y equipos desde etapas tempranas del diseño.

- Permite desarrollar programas de vigilancia y mantenimiento basado en la criticidad. Desventajas:

- Identifica fallas aisladas, no combinadas.

- Se complica en proporción a la mayor complejidad del objeto de análisis (Velasco, s.f.). Método Delphi: Proceso para obtener consenso de opinión en un equipo de expertos. Se parte de una encuesta sobre la materia en discusión, se expresa la opinión de manera individual y anónima, respondiendo a un cuestionario, las personas no se reúnen y consolidan la información y se circula entre todos los expertos quienes responden con acuerdos y desacuerdos y se repite hasta lograr consenso.

Ventajas:

- Intervención de expertos en el tema.

- Genera sentido de propiedad.

Desventajas:

- El trabajo se vuelve intensivo y demanda mucho tiempo para lograr el consenso. (Velasco, s.f.).

Análisis de escenarios: Consiste en identificar riesgos considerando los potenciales problemas que pudiesen presentarse y sus consecuencias. Los escenarios que generalmente se utilizan para reflejar la ocurrencia de los riesgos son: "el mejor caso", "el peor caso" y "el caso previsto".

Ventajas:

- Se pueden realizar predicciones a largo plazo y contemplar una gama de opciones y cómo modificar el curso de acción.

Desventajas:

- Cuando la incertidumbre es muy elevada, algunos de los escenarios se tornan irreales. 
- Los escenarios utilizados para tomar decisiones podrían contener datos especulativos; por lo que, los resultados que se generen no serán realistas (Instituto Nacional de Normalización - INN, 2013).

Herramienta RISCAR: Acosta (como se citó en Mejía, 2006) describe a esta herramienta como un método estructurado y probado que garantiza la efectividad de su aplicación por lo práctica que resulta su implementación.

Ventajas:

- Incluye todas las etapas básicas para la administración de riesgos.

- Garantiza la administración integral de los riesgos.

- Permite la utilización eficiente de los recursos económicos en el tratamiento de los riesgos.

Desventajas:

- Para que este método sea eficaz, es necesario que los responsables estén debidamente capacitados en temas de riesgos.

Método PREST: Se trata de un instrumento que facilita la descripción, a detalle, del entorno dentro del cual la organización se desenvolverá (perfil estratégico). Ayuda a entender el comportamiento de un mercado y propicia la identificación de las dificultades y retos que se pueden presentarse para una organización.

Ventajas:

- Facilita la toma de decisiones a través del conocimiento del mercado y de los factores que determinarán su desarrollo, permitiendo identificar y controlar los riesgos y establecer si es conveniente o no entrar en dicho mercado.

- Facilita la planificación y minimiza el impacto de los escenarios adversos.

Desventajas:

- Se enfoca mayormente en factores externos.

- Necesita que se delimite con precisión el mercado en el que operará la organización (Martín, 2017). 
De lo antes descrito, en la presente investigación se aplicó el cuestionario y la entrevista, como técnicas para la evaluación del control interno en lo referente a la administración de riesgos y a la verificación del cumplimiento de los objetivos de la Institución. Huapaya Capcha (2019), plantea que esto permite desarrollar una gestión de calidad, por cuanto existe evaluación constante del proceso gerencial, permitiéndose tomar correctivos en la medida que se aplican estrategias para el logro de los objetivos organizacionales.

\section{METODOLOGÍA}

La recopilación y análisis de datos se enmarcó en una investigación de carácter mixta, predominando lo cualitativo sobre los elementos cuantitativos, por cuanto se fundamentó teóricamente el control interno con enfoque en riesgos estratégicos y su incidencia en la evaluación del cumplimiento de objetivos; y, se realizó un análisis numérico de datos provenientes de estadísticas y observaciones proyectadas en las planificaciones a fin de conocer los efectos de los factores que intervienen en el cumplimiento de los objetivos. Por su alcance, la investigación fue descriptiva-explicativa, por cuanto en primera instancia, se realizó una descripción de la situación problemática en la Dirección de Planificación de la Universidad de Cuenca, en torno a la evaluación del control interno con enfoque en riesgos estratégicos; $y$, se explicaron los procedimientos para el análisis de las causas y de los efectos generados en los componentes de los riesgos, que intervienen en el cumplimiento de objetivos.

Según la finalidad, fue investigación transversal, puesto que se recopiló, por una sola vez, a través de la aplicación de cuestionarios y entrevistas, la información procedente del universo de estudio.

Así también, los métodos utilizados fueron analítico-sintético, al analizar por separado los componentes, tanto del control interno como de los riesgos estratégicos, a fin de determinar las causas y efectos en la evaluación del cumplimiento de los objetivos; el método inductivo-deductivo, al analizar las variables de estudio desde un marco general de referencia, para posteriormente indagar sobre acontecimientos puntuales generados 
dentro de los procesos de evaluación de control interno con enfoque en riesgos estratégicos, realizados por la Dirección de Planificación.

Además, se apoyó en el método de modelación, con la revisión de formatos estructurales disponibles en la Dirección de Planificación, como planes operativos anuales, a través de los que se revisó el cumplimiento de los planes, programas y proyectos consolidados; y, cuestionarios de evaluación de control interno, diseñados por la Unidad de Auditoría Interna de la Institución, que incluyeron parámetros y calificaciones preestablecidas para determinar el grado de cumplimiento de la normativa y de los procedimientos.

Como complemento, a fin de obtener datos lo más cercanos a la realidad, en esta investigación se aplicaron tres técnicas, como se detalla en la Tabla 1:

Técnicas e instrumentos utilizados en la investigación Tabla 1

Técnicas Instrumentos

\begin{tabular}{ll}
\hline Entrevista & Cuestionario semiestructurado \\
Encuesta & Cuestionario
\end{tabular}

Análisis documental Ficha de contenido

Elaborado por: Grupo de Investigación

Técnica de la encuesta: Utilizada con el propósito de conseguir información a través de un interrogatorio, donde se plantean preguntas con un orden predeterminado, que pueden incluirse en un cuestionario formal (Naresh, 2008).

Esta técnica fue utilizada con la aplicación de preguntas diseñadas para recabar información acerca del cumplimiento de los objetivos de la Universidad; y, de las evaluaciones de control interno con enfoque en riesgos estratégicos, que la Institución aplica.

Técnica de la entrevista: Es una forma de obtener información directa y personal, en la que un entrevistador interroga a una persona con la finalidad de saber sus opiniones, 
conocimientos, motivaciones, actitudes y sentimientos subyacentes acerca del tema de estudio (Naresh, 2008).

Se realizaron entrevistas, por medio de cuestionarios semiestructurados, donde se elaboraron preguntas a modo de guion y su desarrollo fue variando en función de las respuestas recibidas de los entrevistados para la obtención de información más completa. La entrevista se aplicó a la máxima autoridad de la Institución; su Rector, quien a su vez, es el representante superior del Consejo Universitario, encargado de conocer y aprobar los planes estratégicos, planes operativos anuales, informes de rendición de cuentas, entre otros documentos que conciernen al objeto de la investigación.

En igual forma, se realizó una entrevista al Director de Planificación a fin de conocer a profundidad sobre las herramientas utilizadas para evaluar el cumplimiento de objetivos en cada dependencia de la Institución y sobre los riesgos estratégicos a los que están expuestas.

Análisis documental: Constituye una fuente valiosa de datos cualitativos que ayudan al investigador a entender el fenómeno central de estudio, sus antecedentes, situaciones y funcionamiento cotidiano (Hernández, Fernández y Baptista, 2014).

Al respecto, se revisaron documentos formulados por entes de control e instituciones que emiten directrices respecto a temas de la evaluación de control interno y de riesgos, como es el caso de las Normas de Control Interno, de la Contraloría General del Estado; los lineamientos del Plan Estratégico de Desarrollo Institucional y los Planes Operativos Anuales, que reposan en la Dirección de Planificación de la Universidad de Cuenca y contiene importantes datos sobre el cumplimiento de los objetivos institucionales.

\section{Universo de estudio}

La población, cuya característica fue de ejecución de las actividades a ser evaluadas en cuanto a cumplimiento de objetivos, consistió en todas las dependencias y facultades que constituyen la Universidad de Cuenca.

Sin embargo a lo expuesto, la población objeto de estudio se conformó únicamente por el personal que integra la Dirección de Planificación de la Universidad de Cuenca, por 
cuanto, dicha dependencia se encuentra relacionada directamente con el objetivo central de la investigación, al ser la encargada de la coordinación y evaluación del cumplimiento de los objetivos estratégicos.

Bajo esta explicación, la población objeto de estudio se conformó según lo descrito en la siguiente tabla:

\section{Población objetivo}

Tabla 2

Población relacionada con actividades de evaluación de cumplimiento

Dirección de Planificación:

- Director

- Analistas de planificación estratégica y proyectos

- Especialista de información, evaluación y seguimiento

Población total
Total de servidores

Población total 7

Fuente: Planificación de Talento Humano 2018 de la Universidad de Cuenca

\section{RESULTADOS}

Los resultados obtenidos se presentan tanto en valores absolutos como en equivalencias porcentuales; con dichos resultados, se elaboraron figuras de barras a fin de tener una visión rápida de la información generada. La interpretación y análisis se agrupó por tipo de indicador de cumplimiento, debiendo señalar que, las respuestas obtenidas en los cuestionarios, se cotejaron con las respuestas de las encuestas, complementando de esta manera el análisis de los datos. 
A continuación, se presentan los resultados, según lo descrito:

\section{Indicador: Plan de riesgos}

El $71 \%$ de encuestados coincidieron en que no existen mecanismos internos que contribuyan a identificar potenciales riesgos que pudiesen afectar la consecución de los objetivos institucionales.

\section{Existencia de mecanismos internos de identificación de riesgos}

\section{Figura 2.}

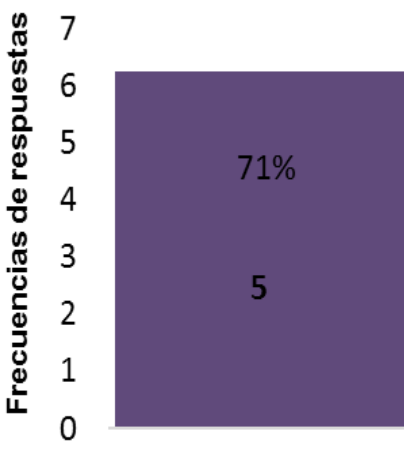

No

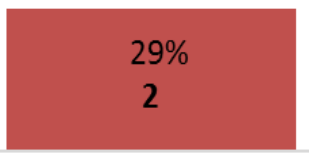

Sí

Alternativas de respuesta

En concordancia con lo descrito, la mayoría de encuestados manifestaron que no existe un plan de riesgos que permita analizar los cambios que pudiesen llegar a afectar la consecución de objetivos (ver figura 3); por ende, el $86 \%$ señaló que tampoco se asignan, en las planificaciones, recursos para ejecutar acciones de respuesta a los riesgos, como se aprecia en la figura 4. 
Existencia de un plan de riesgos.

Figura 3

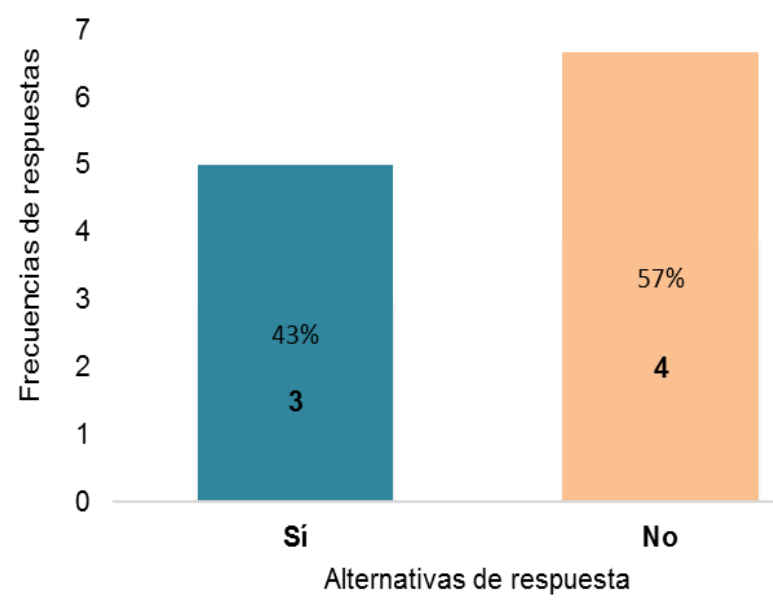

Asignación de recursos para dar respuesta a los riesgos

Figura 4.

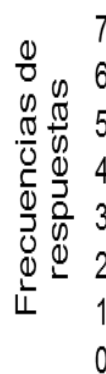

$14 \%$

1

Sí

No
$86 \%$

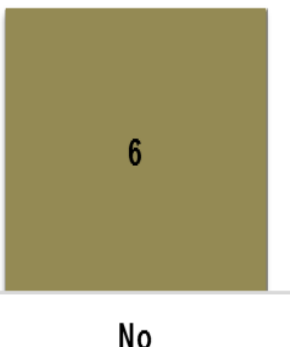

Al respecto, al consultar en la entrevista realizada al Director de Planificación, si existe alguna herramienta para identificar riesgos potenciales que pudiesen impedir el cumplimiento de los objetivos institucionales, manifestó que no existe ningún mecanismo, que únicamente se encuentran en proyección de desarrollo.

\section{Indicador: Documentos y herramientas para determinar objetivos}

Para tener una perspectiva en cuanto a este indicador, se observa en la figura 5, un cumplimiento del 100\%, puesto que todos los encuestados manifestaron que se presentan a la máxima autoridad, de manera periódica, los informes de gestión en los que consta la relación entre los objetivos planificados y lo ejecutado. 
Presentación de informes de gestión periódicos a la máxima autoridad Figura 5

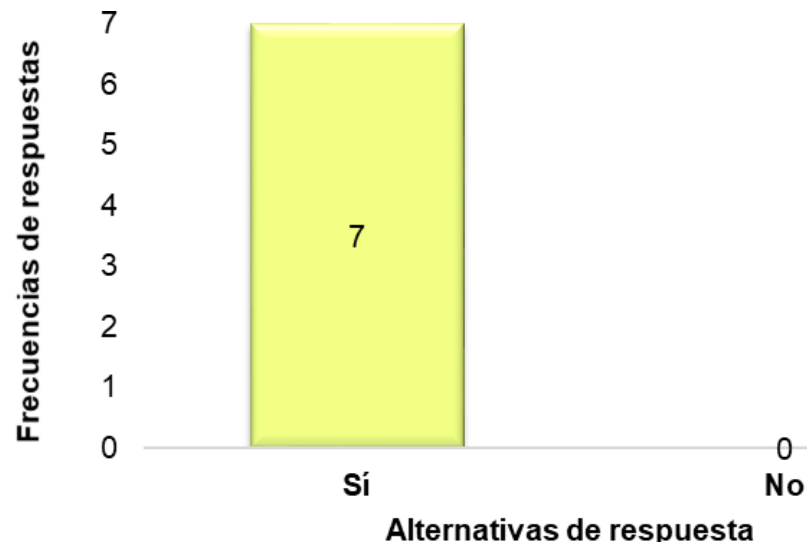

Así mismo, al preguntar a los encuestados sobre los documentos que fueron socializados en la comunidad universitaria, indican que, entre estos documentos, constan los informes de gestión y rendición de cuentas. El 100\% afirma que el documento de planificación estratégica ha sido socializado; no obstante, en cuanto al código de ética, tan solo dos personas tienen conocimiento de que haya sido socializado. 


\section{Documentos socializados en la comunidad universitaria}

Figura 6

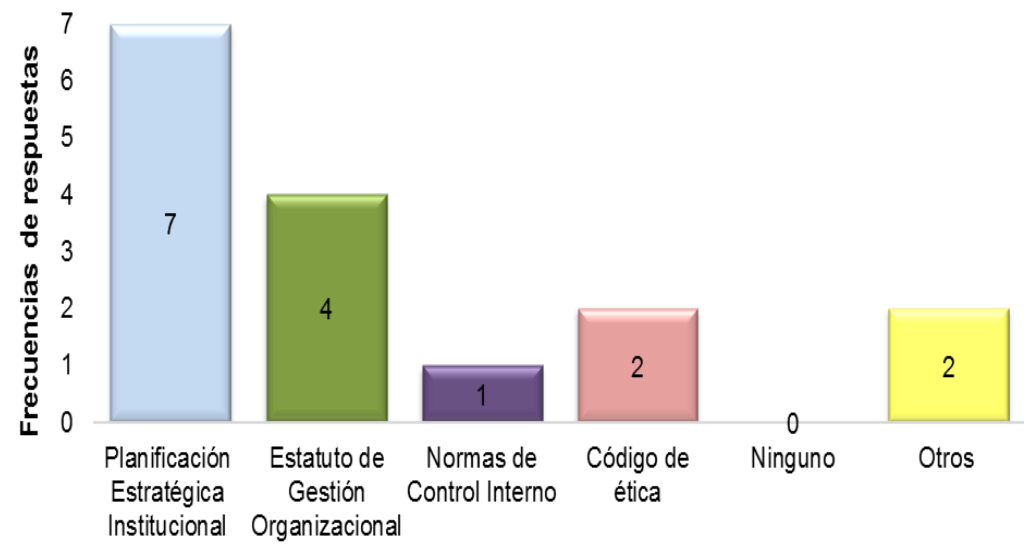

Alternativas de respuesta

En la entrevista efectuada al Director de Planificación, se consultó sobre la existencia de políticas o procedimientos que faciliten la formulación de objetivos en las dependencias universitarias, a lo cual refirió indicando que, en el estatuto institucional y en la normativa emitida por los entes gubernamentales se contemplan las directrices para determinar los objetivos institucionales.

Por otra parte, del análisis documental realizado en el presente estudio, se examinó el plan estratégico de desarrollo institucional 2017-2021 de la Universidad de Cuenca y los informes anuales de rendición de cuentas, como documentos base para la identificación de los objetivos institucionales; los cuales aportaron, además, para recoger los resultados de la gestión institucional en lo que respecta a las evaluaciones de control interno.

\section{Indicador: Control interno}

Como se puede observar en la siguiente figura, en lo que respecta a la existencia de sistemas o mecanismos de control interno que incluyan la formulación, ejecución, control, seguimiento y evaluación de los objetivos institucionales planteados, el $57 \%$ indicó que sí existen mecanismos de control interno; no obstante, el $43 \%$ señaló que no existen tales mecanismos de control, como se observa a continuación. 


\section{Existencia de mecanismos de control interno para evaluación de objetivos}

Figura 7

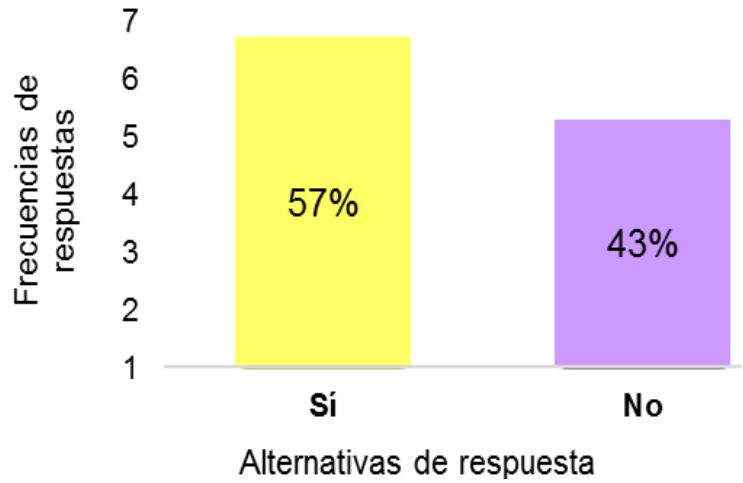

\section{Indicador: Planes Operativos Anuales}

Es importante resaltar que la totalidad de encuestados afirmaron que se elaboran Planes Operativos Anuales en la Institución, en donde se plasman los objetivos a cumplir a corto y largo plazo, como se expone en la figura 8, siendo el factor denominado "presupuesto", el de mayor importancia al momento de definir los objetivos; y, el de menor importancia, el factor "coherencia con la misión y visión institucionales". 
Elaboración de Planes Operativos Anuales

Figura 8

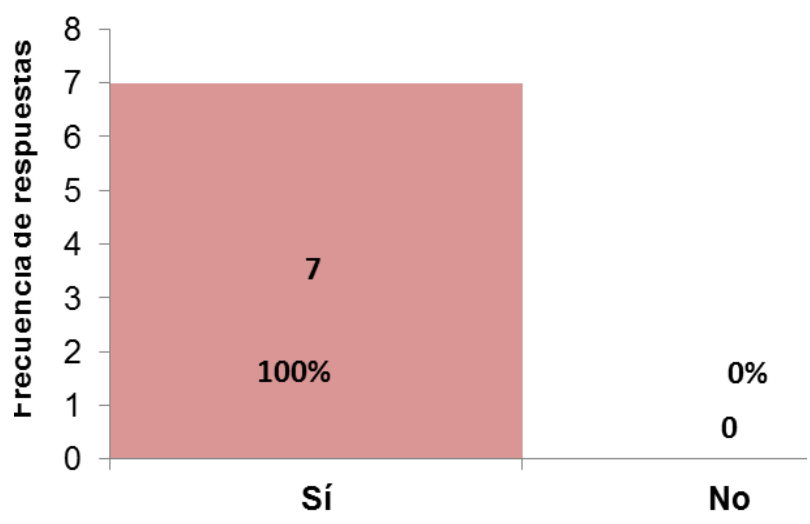

Alternativas de respuesta

Indicador: Evaluaciones de cumplimiento continuas y periódicas

Dentro de este análisis, se obtuvo como resultado que, el 100\% de encuestados, según se presenta en la figura 9, alegaron que los objetivos institucionales establecidos sí poseen métricas de medición y porcentajes del nivel de su cumplimiento.

Existencia de métricas de cumplimiento de los objetivos

Figura 9

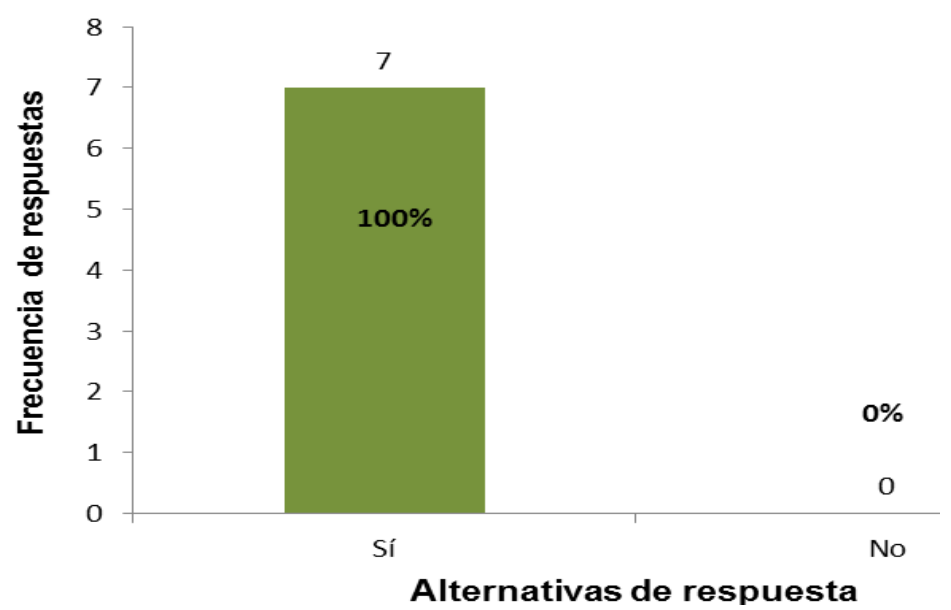


De igual forma, en la figura 10 se puede observar que, el 57\% de encuestados manifestó la no existencia de procesos internos y/o externos estandarizados, de revisión al cumplimiento de objetivos, donde se identifiquen las desviaciones y además se planteen acciones preventivas y correctivas.

\section{Existencia de procesos de revisión de cumplimiento de objetivos} Figura 10.

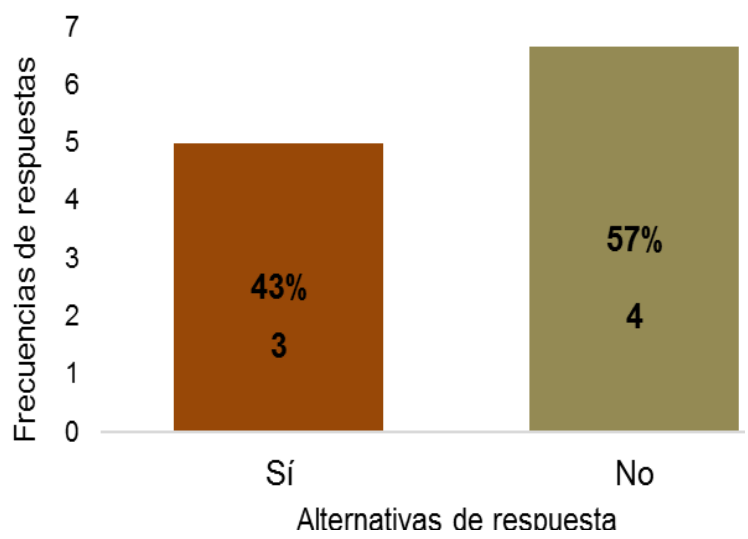

Consecuentemente, en base a los resultados generados, la Dirección de Planificación de la Universidad de Cuenca, no dispone de un sistema de evaluación de control interno para el tratamiento de riesgos estratégicos, que permita identificar los elementos relevantes, tanto de fortalezas como de amenazas para la consecución de objetivos, lo que conlleva a su vez a la inexistencia de mapas de riesgos que faciliten la valoración y formulación de planes de respuesta a dichos riesgos.

\section{CONCLUSIONES}

La creciente complejidad de la realidad institucional conlleva a la Universidad de Cuenca a experimentar dinámicas de cambios inminentes; muchas veces, aparecen simultáneamente conceptos y situaciones que exigen controles más frecuentes y precisos, que deben ser aplicados de manera urgente a fin de alcanzar los objetivos trazados. En este sentido, la Universidad, al ser una entidad que recibe recursos públicos, se somete a las normas, procedimientos y principios determinados por los organismos 
de control y entidades de la administración pública, que regulan, controlan y evalúan el cumplimiento de los objetivos.

Dentro de este orden de ideas, la Asamblea Nacional Constituyente (2008), en el artículo 297, de la Constitución del República del Ecuador, señala que todo programa financiado con recursos públicos tendrá objetivos, metas y un plazo predeterminado para ser evaluado.

Por su parte, la Contraloría General del Estado, en la norma de control interno 100-04, establece que la máxima autoridad, los directivos y demás servidores, de manera periódica, formal y oportuna realizarán la rendición de cuentas sobre el cumplimiento de los objetivos y presentarán informes de su gestión. Así mismo, la norma de control interno 200-02, determina que se deberán implementar sistemas de planificación que incluyan la formulación, ejecución, control, seguimiento y evaluación de los planes institucionales; e, indicadores de gestión para evaluar el cumplimiento de los objetivos.

En el ámbito del sistema educativo, de acuerdo a la Disposición General Quinta de la Ley Orgánica de Educación Superior, las universidades y escuelas politécnicas deberán elaborar planes operativos y planes estratégicos de desarrollo institucional y deberá realizar la evaluación de estos planes.

A partir de este marco regulador, se determinó que la Dirección de Planificación de la Universidad de Cuenca, dispone de herramientas en donde se plasman los objetivos institucionales a corto y largo plazo, como es el caso del plan operativo anual y de la planificación estratégica institucional, que se encuentran alineados a las directrices establecidas por los respectivos organismos de control, ya que, cada unidad ejecutora, en base a parámetros formales emitidos por la Dirección de Planificación y con el acompañamiento de dicha dirección, elaboran las matrices de planificación y de evaluación, de manera periódica, con la inclusión de indicadores de gestión que guardan relación con el cumplimiento de los objetivos institucionales.

En caso de las evaluaciones de cumplimiento de objetivos (ejecución presupuestaria y logro de resultados), estas se realizan de manera parcial, a finales del primer semestre de ejecución de los planes, programas y proyectos, estableciendo los indicadores con 
porcentajes de avance a fin realizar las reformas necesarias para el segundo semestre del año.

Sin embargo; pese a lo indicado, las evaluaciones que se realizan a través de la Dirección de Planificación, no incluyen acciones para gestionar potenciales riesgos estratégicos a los que pudiese estar expuesta la Institución para alcanzar sus objetivos, como dispone para el efecto la norma de control interno 300, de la Contraloría General del Estado, donde señala que la máxima autoridad establecerá los mecanismos necesarios, que incluya la metodología, estrategias, técnicas y procedimientos de identificación, análisis y tratamiento de los riesgos a los que está expuesta la institución.

Por consiguiente, la Dirección de Planificación no dispone de un sistema de evaluación de control interno que incluya el tratamiento de riesgos estratégicos, como lo dispone la Contraloría General del Estado (2003), en el artículo 12 del Reglamento de la Ley Orgánica de la Contraloría General del Estado, que indica que las entidades del sector público serán responsables de implementar y perfeccionar constantemente el sistema de control interno; y que, entre uno de los componentes de control que se aplicarán, será la evaluación de los riesgos.

En el mismo orden de ideas, al no disponer de un mecanismos de análisis y evaluación de riesgos estratégicos, conlleva a la inexistencia de mapas de riesgos que faciliten la valoración y formulación de planes de respuesta, lo que se evidenció en la falta de asignación de recursos en las planificaciones, para hacer frente a los riesgos que llegasen a materializarse. A este respecto, la norma de control interno 300-04, de la Contraloría General del Estado, dispone que los directivos de la institución, analizarán opciones de respuestas al riesgo, tomando en cuenta la relación costo/beneficio.

Como complemento, el artículo 6, literal c), de la Guía Metodológica del GPR, emitido por la de la Secretaría Nacional de Planificación (2011), determina, entre los elementos que conforman los planes estratégicos y operativos institucionales, los siguientes: Objetivo, Indicador y Riesgo; y, en el artículo 8, establece que la etapa de seguimiento y control comprende el ámbito preventivo de riesgos y consiste en su mitigación; es decir, en la reducción de la probabilidad de ocurrencia y del nivel de impacto. 
Atendiendo todas las consideraciones descritas, el trabajo investigativo hace énfasis en la necesidad de que la Dirección de Planificación de la Universidad de Cuenca, como unidad de carácter asesor, técnico y consultivo, encargada de la coordinación del sistema de planificación institucional de direccionamiento estratégico, disponga de un adecuado sistema de control interno, con la incorporación de la gestión de riesgos, que posibilite la detección de desvíos en las programaciones, así como los factores internos y externos a los que la Universidad pudiese estar expuesta para alcanzar sus objetivos, a la vez que incluya la definición de las medidas pertinentes para afrontarlos exitosamente.

\section{REFERENCIAS CONSULTADAS}

1. Acosta, C. (2013). Implementación del Método "RISICAR" para la administración de riesgos en una empresa pequeña. Bogotá, Colombia: Universidad EAFIT.

2. Asamblea Nacional Constituyente. (2008). Constitución de la República del Ecuador.

3. Asociación Española para la Calidad. (2012). Herramientas para grupos de participación. Madrid: Asociación Española para la Calidad.

4. Asociación Española para la Calidad. (2018). Curso Gestión de Riesgos Empresariales. Madrid.

5. Castañeda, I. (n.d.). Gestión de los Riesgos del Proyecto.

6. Castillo, J. C., Erazo, J. C., Narváez, C. I., \& Torres, M. M. (2019). Auditoría de gestión y su incidencia en la eficiencia y eficacia de las operaciones de una empresa comercial. Visionario Digital, 3(2.1.),, 159-188.

7. Comité de Supervisión Bancaria de Basilea. (2003, Febrero). Buenas prácticas para la gestión y supervisión del riesgo operativo. Suiza.

8. Contraloría General del Estado. (2003). Ley Orgánica de la Contraloría General del Estado. Quito.

9. Contraloría General del Estado. (2009, Noviembre 16). Acuerdo 039-CG-2009. Quito. Retrieved from Contraloría General del Estado Web site: http://www.contraloria.gob.ec/Normatividad/BaseLegal 
Revista Arbitrada Interdisciplinaria KOINONIA

Año IV. Vol IV. Nº. Julio - Diciembre 2019

Hecho el depósito de Ley: FA2016000010

ISSN: $2542-3088$

FUNDACIÓN KOINONIA (F.K). Santa Ana de Coro. Venezuela.

Jorge Enrique Sánchez; José Luis Sánchez Espinosa; Jorge Hernando Sánchez Espinosa; Verónica Alexandra Sánchez Espinosa

10. Contraloría General del Estado. (2009, Noviembre 16). Normatividad/BaseLegal/Contraloría General del Estado. Retrieved from Contraloría General del Estado Web site: http://www.contraloria.gob.ec/Normatividad/BaseLegal

11. Delgado, J. (2016, Octubre 18). Que no se te rompa el jarrón: una docena de técnicas para identificar riesgos.

12. El Telégrafo E.P. (2016, Mayo 11). Universidad de Cuenca vuelve a la categoría A. Diario El Tiempo. Retrieved from https://www.eltiempo.com.ec/noticias/cuenca/2/universidad-de-cuenca-vuelve-ala-categoria-a

13. El Telégrafo E.P. (2018, Marzo 24). U. de Cuenca entre las cinco mejores del país. Diario El Tiempo. $\quad$ Retrieved from https://www.eltiempo.com.ec/noticias/cuenca/2/u-de-cuenca-entre-las-cincomejores-del-pais

14. García, J., \& Salazar, P. (2015). Métodos de Administración y Evaluación de Riesgos. Primavera, Chile: Universidad de Chile.

15. García, V. (2014). Introducción a las Finanzas, 2a Edición. México: Grupo Editorial Patria.

16. Gil, F. (2009). Reflexiones sobre la Financiación de los Gobiernos Descentralizados. Cuenca, España: Ediciones de la Universidad de Castilla-La Mancha.

17. Hernández, R., Fernández, C., \& Baptista, M. d. (2014). Metodología de la investigación. Distrito Federal, México: McGraw-Hill.

18. Huapaya Capcha, Y. (2019). Gestión por procesos hacia la calidad educativa en el Perú. Revista Arbitrada Interdisciplinaria Koinonía, 4(8), 243-261. doi:http://dx.doi.org/10.35381/r.k.v4i8.277

19. Instituto Nacional de Normalización - INN. (2013, Marzo 27). Norma Chilena NChISO 31010. Gestión del riesgo-Técnicas de evaluación del riesgo, 1, 27. Chile.

20. Martín, J. (2017, Mayo 15). ESTUDIA TU ENTORNO CON UN PEST-EL. Ecuador.

21. Medina, J., Lascano, N., \& Neyfe, S. (2017). La mitigación de riesgos y su incidencia en el control interno de las Universidades del sector público en el Ecuador. Atlante: Cuadernos de Educación y Desarrollo. 
22. Medina, J., Nelson, L., \& Neyfe, S. (2017). La mitigación de riesgos y su incidencia en el control interno de las Universidades del sector público en el Ecuador. Atlante: Cuadernos de Educación y Desarrollo.

23. Naresh, M. (2008). Investigación de Mercados. México: Pearson Education.

24. Norma Internacional ISO 31000. (2009, Noviembre 15). Norma ISO 31000 versión 2009: Gestión de Riesgos-Principio y Guías. 1.

25. Owen, R. (2014). Riesgo estratégico: La piedra angular para la transformación del riesgo. Deloltte, 6.

26. Rivas, G. (2011). Modelos contemporáneos de control interno. Fundamentos teóricos. Observatorio Laboral, 122-127.

27. Seco, M. (2007, Septiembre). Riesgos económicos y financieros de la empresa. Escuela de Negocios EOI.

28. Secretaría de Educación Superior, Ciencia, Tecnología, Innovación y Saberes Ancestrales. (2018). Educación Superior, Ciencia, Tecnología, Innovación y Saberes Ancestrales en Cifras Diciembre-2018. Quito-Ecuador.

29. Secretaría de Educación Superior, Ciencia, Tecnología, Innovación y Saberes Ancestrales. (2018). Secretaría de Educación Superior, Ciencia, Tecnología, Innovación y Saberes Ancestrales en Cifras Ecuador-Azuay Diciembre 2018. Quito-Ecuador.

30. Secretaría Nacional de Planificación. (2011). Guía Metodológica del GPR.

31.SENESCYT. (2018). Educación Superior, Ciencia, Tecnología, Innovación y Saberes Ancestrales en Cifras Diciembre-2018. Quito-Ecuador.

32. Trespalacios, J., Vázquez, R., \& Bello, L. (2005). Método de recogida y análisi de la información para la toma de decisiones en marketing. Madrid: THOMSON.

33. Universidad de Cuenca. (2018). Planificación de Talento Humano. Planificación de Talento Humano.

34. Universidad de Cuenca. (2018, Julio 25). Universidad de Cuenca Información General. Retrieved from Universidad de Cuenca Información General: https://www.ucuenca.edu.ec/sobre-la-udc/informacion-general 
35. Velasco, A. (n.d.). Claves para la Gestión de Riesgos. Lima, Perú: Universidad de San Martín de Porres.

\section{REFERENCES CONSULTED}

1. Acosta, C. (2013). Implementation of the "RISICAR" Method for risk management in a small company. Bogotá, Colombia: EAFIT University.

2. National Constituent Assembly. (2008). Constitution of the Republic of Ecuador.

3. Spanish Association for Quality. (2012). Tools for participation groups. Madrid: Spanish Association for Quality.

4. Spanish Association for Quality. (2018). Business Risk Management Course. Madrid.

5. Castañeda, I. (n.d.). Project Risk Management.

6. Castillo, J. C., Erazo, J. C., Narváez, C. I., \& Torres, M. M. (2019). Management audit and its impact on the efficiency and effectiveness of the operations of a commercial company. Digital Visionary, 3 (2.1.) ,, 159-188.

7. Basel Committee on Banking Supervision. (2003, February). Good practices for the management and supervision of operational risk. Switzerland.

8. General Contralory of the State. (2003). Organic Law of the Comptroller General of the State. Quito.

9. General Contralory of the State. (2009, November 16). Agreement 039-CG-2009. Quito Retrieved from Comptroller General of the State Website: http://www.contraloria.gob.ec/Normatividad/BaseLegal

10. General Contralory of the State. (2009, November 16). Normatividad / BaseLegal / Comptroller General of the State. Retrieved from Comptroller General of the State Website: http://www.contraloria.gob.ec/Normatividad/BaseLegal

11. Delgado, J. (2016, October 18). Don't let the vase break: a dozen techniques to identify risks.

12. The Telegraph E.P. (2016, May 11). Universidad de Cuenca returns to category A. Diario 
Revista Arbitrada Interdisciplinaria KOINONIA

Año IV. Vol IV. Nº. Julio - Diciembre 2019

Hecho el depósito de Ley: FA2016000010

ISSN: 2542-3088

FUNDACIÓN KOINONIA (F.K). Santa Ana de Coro. Venezuela.

Jorge Enrique Sánchez; José Luis Sánchez Espinosa; Jorge Hernando Sánchez Espinosa; Verónica Alexandra Sánchez Espinosa

https://www.eltiempo.com.ec/noticias/cuenca/2/universidad-de-cuenca-vuelve-ala-categoria-a

13. The Telegraph E.P. (2018, March 24). U. of Cuenca among the five best in the country. El Tiempo newspaper. Retrieved from https://www.eltiempo.com.ec/noticias/cuenca/2/u-de-cuenca-entre-las-cincomejores-del-pais

14. García, J., \& Salazar, P. (2015). Methods of Administration and Risk Assessment. Spring, Chile: University of Chile.

15. García, V. (2014). Introduction to Finance, 2nd Edition. Mexico: Patria Editorial Group.

16. Gil, F. (2009). Reflections on the Financing of Decentralized Governments. Cuenca, Spain: Editions of the University of Castilla-La Mancha.

17. Hernández, R., Fernández, C., \& Baptista, M. d. (2014). Investigation methodology. Federal District, Mexico: McGraw-Hill.

18. Huapaya Capcha, Y. (2019). Management by processes towards educational quality in Peru. Interdisciplinary Arbitrated Review Koinonía, 4 (8), 243-261. doi: http: //dx.doi.org/10.35381/r.k.v4i8.277

19. National Institute for Standardization - INN. (2013, March 27). Chilean Standard NCh-ISO 31010. Risk management-Risk assessment techniques, 1, 27. Chile.

20. Martín, J. (2017, May 15). STUDY YOUR ENVIRONMENT WITH A PEST-EL. Ecuador.

21. Medina, J., Lascano, N., \& Neyfe, S. (2017). Risk mitigation and its impact on the internal control of public sector universities in Ecuador. Atlante: Notebooks of Education and Development.

22. Medina, J., Nelson, L., \& Neyfe, S. (2017). Risk mitigation and its impact on the internal control of public sector universities in Ecuador. Atlante: Notebooks of Education and Development.

23. Naresh, M. (2008). Market research. Mexico: Pearson Education.

24. International Standard ISO 31000. (2009, November 15). ISO Standard 31000 version 2009: Risk Management-Principle and Guidelines. one. 
Revista Arbitrada Interdisciplinaria KOINONIA

Año IV. Vol IV. Nº. Julio - Diciembre 2019

Hecho el depósito de Ley: FA2016000010

ISSN: $2542-3088$

FUNDACIÓN KOINONIA (F.K). Santa Ana de Coro. Venezuela.

Jorge Enrique Sánchez; José Luis Sánchez Espinosa; Jorge Hernando Sánchez Espinosa; Verónica Alexandra Sánchez Espinosa

25. Owen, R. (2014). Strategic risk: The cornerstone for risk transformation. Deloltte, 6.

26. Rivas, G. (2011). Contemporary models of internal control. Theoretical fundament. Labor Observatory, 122-127.

27. Seco, M. (2007, September). Economic and financial risks of the company. EOI Business School.

28. Ministry of Higher Education, Science, Technology, Innovation and Ancestral Knowledge. (2018). Higher Education, Science, Technology, Innovation and Ancestral Knowledge in Figures December-2018. Quito, Ecuador.

29. Ministry of Higher Education, Science, Technology, Innovation and Ancestral Knowledge. (2018). Secretariat of Higher Education, Science, Technology, Innovation and Ancestral Knowledge in Figures Ecuador-Azuay December 2018. Quito-Ecuador.

30. National Secretariat of Planning. (2011). GPR Methodological Guide.

31. SENESCYT. (2018). Higher Education, Science, Technology, Innovation and Ancestral Knowledge in Figures December-2018. Quito, Ecuador.

32. Trespalacios, J., Vázquez, R., \& Bello, L. (2005). Method of collecting and analyzing information for decision making in marketing. Madrid: THOMSON.

33. University of Cuenca (2018). Human Talent Planning. Human Talent Planning.

34. University of Cuenca (2018, July 25). University of Cuenca General Information. Retrieved from University of Cuenca General Information: https://www.ucuenca.edu.ec/sobre-la-udc/informacion-general

35. Velasco, A. (n.d.). Keys to Risk Management. Lima, Peru: University of San Martín de Porres.

C2019 por los autores. Este artículo es de acceso abierto y distribuido según los términos y condiciones de la licencia Creative Commons Atribución-NoComercial-Compartirlgual 4.0 Internacional (CC BY-NC-SA 4.0)

(https://creativecommons.org/licenses/by-nc-sa/4.0/). 